\title{
Erratum to: Weak Convergence of a Mass-Structured Individual-Based Model
}

\author{
Fabien Campillo • Coralie Fritsch
}

Published online: 7 March 2015

(C) Springer Science+Business Media New York 2015

\section{Erratum to: Appl Math Optim DOI 10.1007/s00245-014-9271-3}

The author would like to correct the errors in the Original Publication.

1. Before the end of the Sect. 3.2 "Growth Flow", the following text should be corrected:

$$
\mathbb{M}(\mathcal{X})=\cdots
$$

should be replaced by

$$
\mathcal{M}(\mathcal{X})=\cdots
$$

The online version of the original article can be found under doi:10.1007/s00245-014-9271-3.

F. Campillo $(\bowtie)$

INRIA, Montpellier, France

e-mail: Fabien.Campillo@inria.fr

C. Fritsch

Montpellier 2 University and INRA/MIA, Montpellier, France

e-mail: Coralie.Fritsch@supagro.inra.fr

F. Campillo · C. Fritsch

MODEMIC Project-Team, INRA/INRIA, UMR MISTEA, 2 Place Pierre Viala,

34060 Montpellier Cedex 01, France 
2. The following text should be corrected of Proposition 4.6:

- Last line of the Proposition 4.6:

$$
\ldots \text { and } v \in \mathcal{M}_{F}(\mathcal{X}) \text {. }
$$

should be replaced by

$$
\ldots \text { and } v \in \mathcal{M}(\mathcal{X}) \text {. }
$$

- Under Proposition 4.6 first line of proof:

$$
\ldots \text { and } v_{0}=v \in \mathcal{M}_{F}(\mathcal{X}) \text {. }
$$

should be replaced by

$$
\ldots \text { and } v_{0}=v \in \mathcal{M}(\mathcal{X}) \text {. }
$$

- Under Proposition 4.6 suppress the second line of proof:

$$
\text { First we suppose that } v \in \mathcal{M}(\mathcal{X}) \text {. }
$$

- Page 18, suppress the last paragraph of the proof.

3. Remark 5.6, third line from the bottom: something could be added after " $f \in$ $C^{1}(\mathcal{X})$." that is, replace:

and $f \in C^{1}(\mathcal{X})$. Note that this generator has the same "substrat" part than that of the initial generator (12) which again justifies the Remark 5.1.

by

and $f \in C^{1}(\mathcal{X}) ; s \in \mathbb{R}_{+}$and $\nu \in \mathcal{M}^{n}(\mathcal{X})=\left\{\frac{1}{n} \Sigma_{i=1}^{N} \delta_{x^{i}}, ; N \in \mathbb{N}, x^{i} \in \mathcal{X}\right\}$. Note that this generator has the same "substrat" part than that of the initial generator (12) which again justifies the Remark 5.1. 